\title{
13 Physical function, quality of life and energy expenditure during activities of daily living in post-bariatric surgery patients and obese individuals
}

\author{
Fabiane Monteiro ${ }^{1}$, Diego Augusto Nascimento Ponce ${ }^{1}$, Humberto Silva ${ }^{1}$, Fábio Pitta ${ }^{1}$, Alexandre J. F. Carrilho ${ }^{2}$ \\ 1- Laboratory of Research in Respiratory Physiotherapy, Department of Physiotherapy, State University of Londrina (UEL), Londrina, Brazil \\ 2- Endocrinology Service, Health Sciences Center, State University of Londrina (UEL), Londrina, Brazil
}

\section{OBJECTIVES}

Bariatric surgery (BS) is an effective method to weight loss, however, some patients experience persistence of physical inactivity. Furthermore, It is not clear if some surgical techniques, mainly those with some disabsorptive components, could be involved in the low physical performance through interfering with nutritional status. This study aimed to evaluate physical function (PF), quality of life $(Q O L)$ and energy expenditure (EE) during activities of daily living (ADL) in post-BS patients and compare with severely obese individuals not submitted to BS.

\section{METHODS}

Forty two subjects were included in the study: 21 post-BS patients ( 3 to 4 years post Roux-en-Y gastric bypass technique) with stable weight for at least 6 months (group PO); and 21 obese individuals without BS (group OB). PF was estimated by the Glittre ADL-test (1), a standardized test which uses ADL. These activities involve rising from a chair, lifting, carrying and bending. EE was assessed by the multisensor sensewear armband activity monitor during $A D L$, the body composition was determined by bioelectrical impedance and QOL by the SF-36 questionary (2). In order to access PF and EE in each circuit activity, subjects were submitted to a modified version of the Glittre ADL-test (3). It included the same five activities described above, but performed during 2 minutes each: walking on the level, walking on the level carrying a backpack (10\% of their body weight), rising from a chair and sitting in another chair positioned 1 meter across, walking up/downstairs ( 2 steps) and moving an object weighting $1 \mathrm{~kg}$ from shelves.

\section{RESULTS}

Table 2. Comparison of energy expenditure (in average) during the 5 activities of the Modified Glittre ADL-Test between obesity (OB) and post-operative (PO) groups.

Table 1. Patients characteristics and anthropometric profile of obesity (OB) and post-operative (PO) groups.

\begin{tabular}{lccc} 
Characteristics & $\begin{array}{c}\mathrm{OB} \\
(\mathrm{n}=21)\end{array}$ & $\begin{array}{c}\mathrm{PO} \\
(\mathrm{n}=21)\end{array}$ & $P$ \\
\hline Age & $44 \pm 9$ & $41 \pm 11$ & 0.48 \\
Gender (F/M) & $16 / 5$ & $16 / 5$ & 1 \\
Weight $(\mathrm{Kg})$ & $113 \pm 21$ & $75 \pm 14$ & $<0.01$ \\
BMI $\left(\mathrm{Kg} \cdot \mathrm{m}^{-2}\right)$ & $44 \pm 6$ & $28 \pm 4$ & $<0.01$ \\
Fat mass $(\%)$ & $44 \pm 7$ & $31 \pm 6$ & $<0.01$ \\
Fat mass $(\mathrm{Kg})$ & $50 \pm 13$ & $23 \pm 7$ & $<0.01$ \\
Fat free mass $(\mathrm{Kg})$ & $62 \pm 15$ & $52 \pm 10$ & $<0.01$ \\
Metabolic rate $(\mathrm{Kcal})$ & $1936 \pm 434$ & $1565 \pm 318$ & $<0.01$
\end{tabular}

Data are expressed as mean \pm standard deviation. Student $T$ test $\mathrm{F}=$ female, $\mathrm{M}=$ male, $\mathrm{BMI}=$ body mass index.

\begin{tabular}{|c|c|c|c|}
\hline Activities & $\begin{array}{c}\text { OB } \\
(n=21)\end{array}$ & $\begin{array}{c}P O \\
(n=21)\end{array}$ & $P$ \\
\hline Walking & $28 \pm 7$ & $32 \pm 6$ & 0.06 \\
\hline Walking with a backpack (cal) & $29 \pm 6$ & $31 \pm 6$ & 0.08 \\
\hline Up/Downstairs (cal) & $21 \pm 5$ & $30 \pm 6$ & $<0.01$ \\
\hline Rising/Sitting in chair (cal) & $30 \pm 7$ & $40 \pm 7$ & $<0.01$ \\
\hline Moving objects in shelves (cal) & $22 \pm 8$ & $30 \pm 7$ & $<0.01$ \\
\hline
\end{tabular}

Data are expressed as mean \pm standard deviation. Student $T$ test

Table 3. Differences in quality of life scores (SF-36) between obesity (OB) and post-operative (PO) groups.

\begin{tabular}{lcc|c} 
SF-36 Domains & OB & PO & $\boldsymbol{P}$ \\
Physical Functioning & $52 \pm 23$ & $80 \pm 23$ & $<0.01$ \\
Role-Physical & $54 \pm 32$ & $88 \pm 22$ & $<0.01$ \\
Body Pain & $47 \pm 26$ & $70 \pm 25$ & $<0.01$ \\
General Health & $54 \pm 19$ & $84 \pm 15$ & $<0.01$ \\
Vitality & $48 \pm 19$ & $72 \pm 21$ & $<0.01$ \\
Social Functioning & $55 \pm 28$ & $110 \pm 21$ & $<0.01$ \\
Role-Emotional & $48 \pm 42$ & $84 \pm 33$ & $<0.01$ \\
Mental Health & $55 \pm 28$ & $78 \pm 19$ & $<0.01$ \\
Data are expressed as mean \pm standard deviation. Student T test &
\end{tabular}

\section{CONCLUSIONS}

Post-bariatric surgery patients have better physical function, quality of life and perform activities under lower total EE than obese subjects. In these subjects, better physical function is associated with better body composition and lower EE during activities of daily living.

\section{References}

1. Skumlien $S$, Hagelund T, Bjortuft $O$, Ryg MS. A field test of functional status as performance of activities of daily living in COPD patients. Respir Med 2006; 100/2: 316-323

2. Ciconelli RM, Ferraz MB, Santos W, Meinao I, Quaresma MR. Tradução para a língua portuguesa e validação do questionário genérico de avaliação de qualidade de vida Medical Outcomes Study 36-item Short Form Health Survey SF-36. (Brasil SF-36). Rev Bras Reumatol. 39[3], 143-150. 1999.

3. Cavalheri V, Donaria L, Ferreira T, Finatti M, Camillo CA, Cipulo Ramos EM et al. Energy expenditure during daily activities as measured by two motion sensors in patients with COPD. Respir Med 2011; 105/6: 922-929. 